\title{
RECETAS BOYACENSES ELABORADAS CON PAPAS NATIVAS COLOMBIANAS
}

\section{BOYACENSES RECIPES ELABORATED WITH NATIVE COLOMBIAN POTATOES}

\author{
Quilaguy A. Diana M. y Chaves C. Julian A.* \\ Fundación Universitaria del Área Andina, Programa profesional en Gastronomía y Culinaria. Facultad de Diseño, Comunicación y \\ Bellas Artes. Grupo de Investigación Proyecta. Correos electrónicos: dquilaguy@areandina.edu.co y \\ jchaves12@areandina.edu.co*. Calle 71 \#13-21, Bogotá, Colombia
}

Recibido agosto 12 de 2016; Aceptado noviembre 25 de 2016

\section{RESUMEN:}

La papa es uno de los productos agrícolas de alto consumo en el territorio colombiano y cuenta con una amplia gama de variedades, de las cuales solo pocas son comercializadas, consumidas o conocidas por la población en general. En el departamento de Boyacá, son cultivadas diferentes variedades de papas nativas y las personas de la región elaboran variadas recetas con este producto, lamentablemente estas no están muy difundidas en el país y muchas de estas papas son desaprovechadas por los agricultores y personas de la zona. Pensando en esto, investigadores de la Fundación Universitaria del Área Andina en alianza con la Corporación Colombiana de Investigación Agropecuaria - Corpoica, desarrollaron la presente 
Autor a quien debe dirigirse la correspondencia: Chaves C. Julian A.

${ }^{*}$ Correo electrónico: investigación, la cual tuvo como objetivo rescatar las recetas tradicionales elaboradas con papas nativas colombianas en el Departamento de Boyacá. Para esto, se realizaron unos talleres con habitantes de los municipios de Jenesano, Motavita, Toca y Ventaquemada, durante los cuales los participantes prepararon diferentes platos. A medida que se iban preparando las recetas, docentes y estudiantes entrevistaron a las personas y tomaron registro de cada uno de los platos. Como resultado, se pudo detallar la preparación de 15 diferentes platos, en los cuales, las papas nativas que se cultivan en la zona, fueron incluidas como ingrediente principal. También se logró evidenciar que recetas denominadas bajo el mismo nombre pueden variar en su preparación según el municipio. Con el propósito de difundir estas recetas y potencializar el uso de las papas nativas de la región, estas preparaciones fueron divulgadas en un recetario.

Palabras clave: Culinaria, gastronomía, papas nativas y receta.

\section{ABSTRACT}

The potato is a highly consumed agricultural product in the Colombian territory and it has a wide range of varieties, of which only few are commercialized, consumed or known by the population in general. In the department of Boyacá, different varieties of native potatoes are cultivated and the people of the region use varied recipes with this product, unfortunately these are not very widespread in the country and many of these potatoes are wasted by the farmers and people of the zone. Thinking about this, researchers from the Fundación Universitaria del Área Andina in alliance with the Corporación Colombiana de Investigación Agropecuaria Corpoica, developed the present investigation, which aims to 
rescue the traditional recipes elaborated with Colombian native potatoes in the Department of Boyacá. For this, workshops were held with inhabitants of the towns of Jenesano, Motavita, Toca and Ventaquemada, during which the participants prepared different dishes. As the recipes were being prepared, teachers and students interviewed the people and took record of each of the dishes. Thus, it was possible to detail the preparation of 15 different dishes, in which the native potatoes that are grown in the area, were included as the main ingredient. It was also demonstrated that recipes named under the same name may vary in their preparation according to the municipality. To disseminate these recipes and promote the use of native potatoes, these preparations were disclosed in a recipe book.

Key words: Culinary, gastronomy, native potatoes and recipe.

\section{INTRODUCCIÓN}

La papa es uno de los principales productos agrícolas en Colombia, su cultivo se encuentra a lo largo del país y en cada región sobresalen ciertas variedades, las cuales se preparan y consumen de diferentes formas. Para el año 2016, la producción de papa en Colombia alcanzó las 126.100 hectáreas sembradas, concentrándose su cultivo en ocho departamentos, entre los cuales se incluye el Departamento de Boyacá (Riascos, s.f). Como datos de interés cabe mencionar que en Colombia, el sector papero contribuye con el $3,3 \%$ en el producto Interno Bruto agropecuario del país; cerca de 100.000 familias cultivan este tubérculo el cual genera anualmente un estimado de 264.000 empleos directos e indirectos; y más del $90 \%$ de la papa comercializada se consume en estado fresco y otro tanto por la industria de procesamiento. Según el Ministerio de Agricultura y Desarrollo Social (2016), entre los años 1998 al 2016, la producción de papa en Colombia aumentó en un $8 \%$ pese a que el área cultivada disminuyó en un $11 \%$; en muchos casos, esta producción se ha visto afectada por condiciones climáticas como sequías y heladas. Debido a la disminución 
del consumo de papa y al aumento progresivo en los rendimientos, el área sembrada tiende a ser menor. Con respecto al departamento de Boyacá, se estima que el área cultivada alcanza las 34.100 hectáreas y que el $95 \%$ de la producción se destina para consumo en fresco. En cuanto al consumo de papa en Colombia, se ha reportado disminución en su consumo per cápita debido a los cambios que se han presentado en los hábitos de alimentación y a la competencia con otros productos (Ministerio de Agricultura y Desarrollo Social, 2016).

Diferentes variedades de papas nativas se cultivan en Colombia, las cuales se presentan en diferentes tamaños, formas y colores, pero no son muy conocidas por los consumidores ya que son poco comercializadas. Estas papas han venido ganando cierto interés gracias a lo agradable de sus propiedades organolépticas como el sabor y la textura; también por sus características nutricionales, pues aportan cantidades importantes de nutrientes y compuestos funcionales, presentan alto contenido de hierro, zinc, potasio, cobre, polifenoles y carotenos $y$ son fuente de energía, proteínas, fibra y minerales (Monteros et al., 2011). Adicional a esto, las papas nativas presentan una ventaja frente a otras por su tolerancia a condiciones ambientales adversas, pues gracias a sus características agronómicas y fisiológicas, las plantas son capaces de tolerar condiciones difíciles como las bajas temperaturas (Gutiérrez, 2008).

En Colombia el germoplasma de papa está conservado en la Colección Central Colombiana de Papa (CCC) la cual alberga especies nativas, introducidas y silvestres; y cuenta con cerca de 3.000 accesiones que comprende diferentes especies como Solanum tuberosum (subespecies andígena y tuberosum); S. chaucha, S. phureja; y especies silvestres como $S$. colombianum y S. estradae (Moreno \& Valbuena, 2006). Para el caso del departamento de Boyacá, se pueden encontrar diferentes variedades de papas nativas como son Tucarreña, Arbolona negra, Pastusa palinegra, Pastusa blanca y Argentina colorada (entre otras) (Moreno et al., 2010).

Las variedades de papas nativas conservadas en los bancos de germoplasma de Colombia, son base de investigaciones gracias a sus cualidades culinarias. Como resultado de los estudios se pueden definir las variedades de papa según características como el sabor, color, harinosidad, humedad y consistencia del tubérculo, y el agrado o no al paladar del consumidor, se relaciona con características que se describen en los perfiles sensoriales del producto (Monteros et al., 2011); (Navarro et al., 2013). 
Para países como Colombia, las papas nativas son material promisorio para enfrentar la crisis alimentaria y se ven como alternativa para sobrepasar los desafíos de producción a causa del cambio climático. A pesar de sus beneficios, las papas nativas han dejado de ser cultivadas debido a la inserción de nuevos materiales comerciales, lo cual ha afectado la conservación de este recurso genético tan importante para el país (Lasso, s.f.).

La papa es uno de los tubérculos preferidos en la cocina colombiana y a la hora de prepararla, es importante considerar qué tipo de papa conviene usar según el plato que se desea; estas pueden ser preparadas de diversas formas como hervidas, al horno, fritas o asadas, entre otras; y son usadas ampliamente en la gastronomía colombiana para preparar diferentes platos (Monteros et al., 2011); (Rodríguez et al., 2013). Una de las tendencias gastronómicas de la actualidad, es regresar a lo básico, a lo heredado de los antepasados, a lo originario de cada país, por lo cual, los platos nacionales están tomando fuerza y alrededor del mundo se está buscando rescatar las raíces, recetas e ingredientes autóctonos de cada región; la tendencia de regresar a la tradición produce una sensación de pertenencia a un lugar, grupo o a una familia (Hernández et al., 2016). La cocina es patrimonio cultural, hace parte de la historia de una comunidad y genera un sentido simbólico y un valor significativo para cocineros y consumidores; las preparaciones tienen historia por su significado y por los recuerdos y memorias que representan para los consumidores; el patrimonio gastronómico se conserva considerando el valor histórico de los platos del pasado (Mejía et al., 2014).

Considerando lo anteriormente mencionado, investigadores del programa profesional en Gastronomía y Culinaria de la Fundación Universitaria del Área Andina y del Plan de Vinculación de la Red de Raíces y Tubérculos - Sistema Papa, que ejecuta la Corporación Colombiana de Investigación Agropecuaria - Corpoica, llevaron a cabo la presente investigación la cual tuvo como objetivo rescatar las recetas tradicionales elaboradas con papas nativas colombianas en el Departamento de Boyacá. Varios esfuerzos ya han sido realizados en pro de la utilización de las papas nativas e incluso existen recetarios donde se han incluido nuevas recetas, pero en este caso se ha querido dar un valor especial a las preparaciones realizadas específicamente en el departamento de Boyacá, con el fin de promover su consumo e incentivar su comercialización. 


\section{MATERIALES Y MÉTODOS}

Para recabar la información de las recetas, se seleccionaron los municipios de Jenesano, Motavita, Toca y Ventaquemada, según los contactos que previamente tenía establecido el personal de Corpoica con personas del departamento de Boyacá. Se realizaron salidas de campo a los municipios mencionados, una salida por día. Para cada una de las salidas, el personal de Corpoica convocó a diferentes personas de cada uno de los municipios, donde se les invitó a participar en unas jornadas relacionadas con el tema de las papas nativas. Los asistentes a estas jornadas fueron principalmente amas de casa, agricultores y personas que pertenecían a agremiaciones de papa de la región.

En cada municipio, el personal de Corpoica organizó el espacio necesario para la preparación de las recetas en lugares como hoteles, fincas o salones de eventos, en donde se contaba con estufas e implementos de cocina con los cuales realizar las preparaciones. Para la elaboración de las recetas, se contó con las siguientes variedades de papas nativas: Argentina, Criolla manzana, Pacha negra, Corneto morado, Calavera, Mambera y Tornilla (ver figura 1).

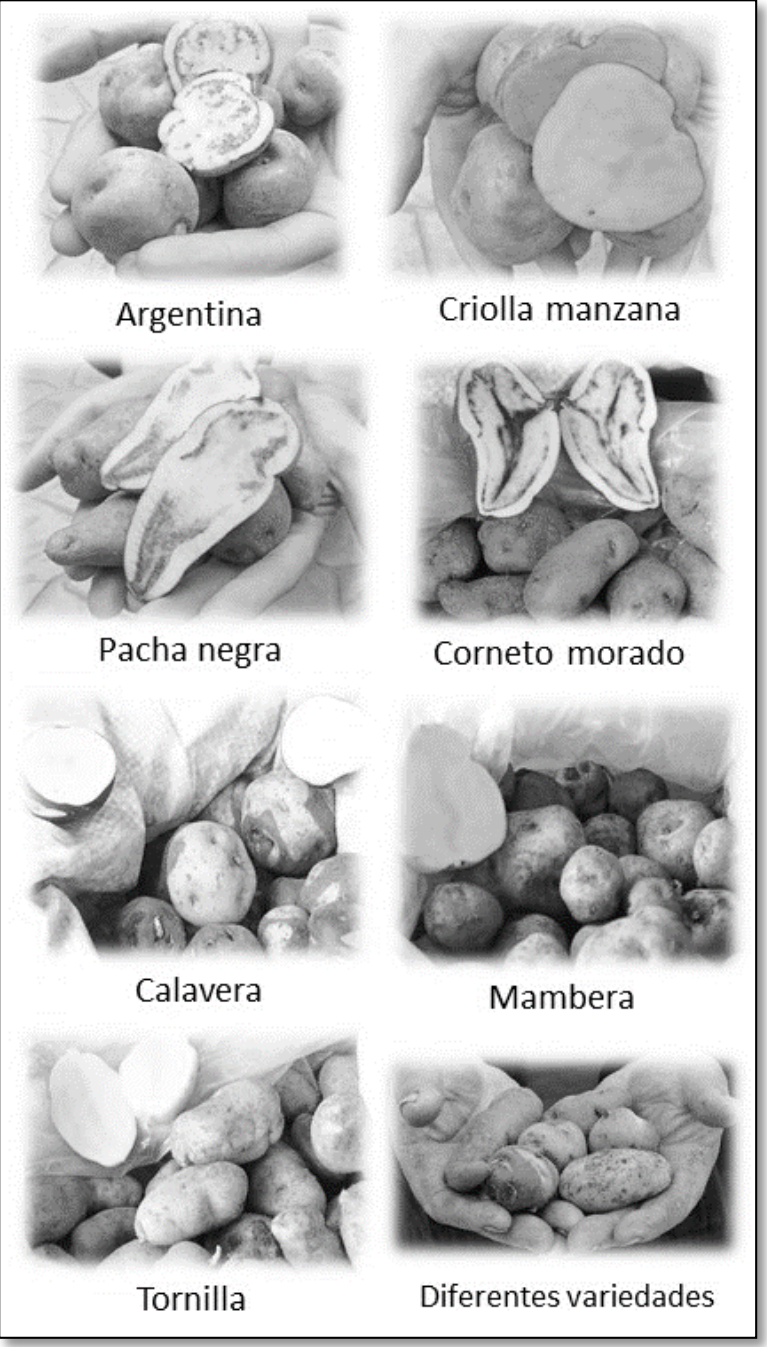

Figura 1. Papas nativas utilizadas para la elaboración de las recetas.

Durante los talleres, los participantes se organizaron en grupos de tres a cinco personas y en la mayoría de los grupos había una persona quien lideraba la elaboración de la receta. Antes de comenzar a cocinar, las personas fueron informadas sobre el objetivo de la investigación y se les solicitó firmar un 
consentimiento informado y la autorización para publicar y divulgar su receta. Cada grupo contó con un promedio de 2 a 3 horas para realizar sus preparaciones. Durante este tiempo, docentes y estudiantes del programa profesional en Gastronomía y Culinaria de la Fundación Universitaria del Área Andina, realizaron las entrevistas a los grupos, diligenciaron el cuestionario preparado para la recolección de la información y tomaron registro de las preparaciones utilizando celulares, una grabadora de voz y una cámara fotográfica.

La mayoría de las papas utilizadas en las preparaciones fueron cocinadas con piel y con técnicas de cocción húmedas con tiempos controlados. Una vez culminaba la preparación de los platos, estos eran degustados con una previa explicación de cada uno de ellos y algunas apreciaciones gastronómicas por parte de los docentes. Las porciones preparadas por cada uno de los platos no fueron estandarizadas, sino que al momento de la degustación, se sirvió una única porción que alcanzara para que los participantes probaran un poco de cada preparación. Teniendo en cuenta que la idea de este ejercicio era conocer las recetas, el proceso de degustación no se realizó con el ánimo de evaluar los platos, sino con la intención de conocer las apreciaciones de los asistentes a los talleres.

Posterior a las salidas de campo, la información registrada en los cuestionarios fue transcrita y organizada para ser publicada a manera de recetario, complementando la información con el registro de voz y fotografías tomadas durante las jornadas de trabajo.

\section{RESULTADOS Y DISCUSIÓN}

Con la presente investigación se logró evidenciar la gran variedad de preparaciones, técnicas y procedimientos que se realizan en algunos de los municipios de Boyacá, en los cuales utilizan las papas nativas como ingrediente principal; muchos de estos platos se han ido perdiendo con el tiempo y otros aún, son preparados por algunos de los habitantes de este departamento. Muestra de esto, son las 15 preparaciones que se lograron documentar a partir de la información recolectada en las salidas de campo (ver tabla 1 y figura 2). 
Tabla 1. Recetas elaboradas e ingredientes utilizados.

\begin{tabular}{|c|c|c|}
\hline $\begin{array}{l}\text { NOMBRE } \\
\text { DE LA } \\
\text { RECETA }\end{array}$ & $\begin{array}{l}\text { PAPAS } \\
\text { NATIVAS }\end{array}$ & DEMÁS INGREDIENTES \\
\hline $\begin{array}{l}\text { Cocido } \\
\text { boyacens } \\
\text { e de } \\
\text { Jenesan } \\
\text { o }\end{array}$ & $\begin{array}{l}\text { - Argentina } \\
\text { - Corneto } \\
\text { rojo } \\
\text { - Criolla } \\
\text { manzana }\end{array}$ & $\begin{array}{l}\text { Cebolla larga y cabezona, } \\
\text { rubas o chuguas, ajo, } \\
\text { mazorcas, tomate, comino, } \\
\text { laurel, tomillo, pimienta, } \\
\text { carne de res, leche, queso } \\
\text { campesino y sal. }\end{array}$ \\
\hline $\begin{array}{l}\text { Cocido } \\
\text { boyacens } \\
\text { e de } \\
\text { Toca }\end{array}$ & $\begin{array}{l}\text { - Argentina } \\
\text { - Criolla } \\
\text { manzana }\end{array}$ & $\begin{array}{l}\text { Tomate, cebolla larga, } \\
\text { nabos, habas, carne de res, } \\
\text { aceite, mazorcas, sal y } \\
\text { leche. }\end{array}$ \\
\hline $\begin{array}{l}\text { Cocido } \\
\text { boyacens } \\
\text { e de } \\
\text { Ventaque } \\
\text { mada }\end{array}$ & - Argentina & $\begin{array}{l}\text { Cubios amarillos, } \\
\text { mazorcas, carne de res, } \\
\text { rubas, sal, habas verdes, } \\
\text { ajos, cebolla larga y } \\
\text { cabezona, tomate, } \\
\text { mantequilla, queso doble } \\
\text { crema, leche y aceite. }\end{array}$ \\
\hline $\begin{array}{l}\text { Ajiaco } \\
\text { motavite } \\
\text { ño }\end{array}$ & $\begin{array}{l}\text { - Argentina } \\
\text { - Pacha } \\
\text { negra } \\
\text { - Criolla } \\
\text { manzana }\end{array}$ & $\begin{array}{l}\text { Carne de res, zanahorias, } \\
\text { cebolla larga, alverja, } \\
\text { lechuga, mazorcas, habas } \\
\text { verdes, ahuyama, nabos } \\
\text { amarillos, plátanos verdes, } \\
\text { fríjoles, cilantro y sal. }\end{array}$ \\
\hline $\begin{array}{l}\text { Ajiaco } \\
\text { indígena }\end{array}$ & $\begin{array}{l}\text { - Pacha } \\
\text { negra } \\
\text { - Criolla } \\
\text { manzana } \\
\text { - Corneto } \\
\text { morado }\end{array}$ & $\begin{array}{l}\text { Ají picante, pimentón, } \\
\text { cebolla ocañera, hojas de } \\
\text { helecho, sal, cilantro, } \\
\text { cilantrón y limón. }\end{array}$ \\
\hline $\begin{array}{l}\text { Sancoch } \\
\text { o } \\
\text { boyacens } \\
\text { e }\end{array}$ & $\begin{array}{l}\text { - Criolla } \\
\text { manzana }\end{array}$ & $\begin{array}{l}\text { Cubios o nabos, habas, } \\
\text { zanahorias, cebolla larga, } \\
\text { mazorcas, sal, carne de } \\
\text { pollo y de res, arveja, frijol, } \\
\text { cilantro, ajo y aguacate. }\end{array}$ \\
\hline $\begin{array}{l}\text { Piquete } \\
\text { boyacens } \\
\text { e de } \\
\text { doña } \\
\text { Amparo, } \\
\text { de la } \\
\text { plaza de } \\
\text { Ventaque } \\
\text { mada } \\
\end{array}$ & Mambera & $\begin{array}{l}\text { Acelgas, mazorcas, } \\
\text { chuguas, tomillo, laurel, } \\
\text { pimienta, cebolla, ajo, } \\
\text { tomate, sal, cilantro, } \\
\text { mantequilla, natas de leche } \\
\text { fresca, queso campesino, } \\
\text { apio, perejil y carne de } \\
\text { ternera. }\end{array}$ \\
\hline
\end{tabular}

\begin{tabular}{|c|c|c|}
\hline $\begin{array}{l}\text { NOMBRE } \\
\text { DE LA } \\
\text { RECETA }\end{array}$ & $\begin{array}{l}\text { PAPAS } \\
\text { NATIVAS }\end{array}$ & DEMÁS INGREDIENTES \\
\hline $\begin{array}{l}\text { Piquete } \\
\text { campesino } \\
\text { de la vereda } \\
\text { de Bojirque, } \\
\text { Ventaquema } \\
\text { da }\end{array}$ & $\begin{array}{l}\text { - Calavera } \\
\text { - Tornilla }\end{array}$ & $\begin{array}{l}\text { Rubas, costilla de res, carne } \\
\text { de ovejo, carne de curí, } \\
\text { ibias, cubios amarillos, } \\
\text { arracachas, mazorcas, } \\
\text { orégano, sal, ajo, pimienta, } \\
\text { laurel, tomillo, cebolla larga y } \\
\text { cuajada fresca. }\end{array}$ \\
\hline $\begin{array}{l}\text { Papa con } \\
\text { cuajada }\end{array}$ & - Argentina & $\begin{array}{l}\text { Tomate, cebolla larga, } \\
\text { leche, cuajada, mantequilla, } \\
\text { sal y perejil. }\end{array}$ \\
\hline $\begin{array}{l}\text { Papa salada } \\
\text { con carne, } \\
\text { con ají de } \\
\text { aguacate }\end{array}$ & - Argentina & $\begin{array}{l}\text { Cebolla, sal, aguacate, ají y } \\
\text { carne de res. }\end{array}$ \\
\hline Puré de papa & $\begin{array}{l}\text { - Criolla } \\
\text { manzana } \\
\text { - Pacha negra }\end{array}$ & $\begin{array}{l}\text { Mazorcas, cebolla, crema de } \\
\text { leche, pollo, carne de res, } \\
\text { arveja, cilantro, aguacate, } \\
\text { tomate, huevos y mayonesa. }\end{array}$ \\
\hline $\begin{array}{l}\text { Ensalada fría } \\
\text { de papa } \\
\text { Argentina }\end{array}$ & - Argentina & $\begin{array}{l}\text { Cebolla larga, tomates, } \\
\text { queso o cuajada, pechuga } \\
\text { de pollo, arveja, aguacate, } \\
\text { mayonesa, lechuga, sal y } \\
\text { ajo. }\end{array}$ \\
\hline Lapingachos & - Chaucha & $\begin{array}{l}\text { Cebolla larga, queso } \\
\text { campesino, achiote, ajo, sal } \\
\text { y empella de cerdo. }\end{array}$ \\
\hline $\begin{array}{l}\text { Arepas de } \\
\text { papa }\end{array}$ & $\begin{array}{l}\text { - Argentina } \\
\text { - Pacha negra }\end{array}$ & $\begin{array}{l}\text { Harina de trigo, queso } \\
\text { campesino, mantequilla, sal } \\
\text { y azúcar. }\end{array}$ \\
\hline Canos & - Argentina & $\begin{array}{l}\text { Harina de maíz tostado, sal } \\
\text { y panela. }\end{array}$ \\
\hline
\end{tabular}

Fuente: Autores. 


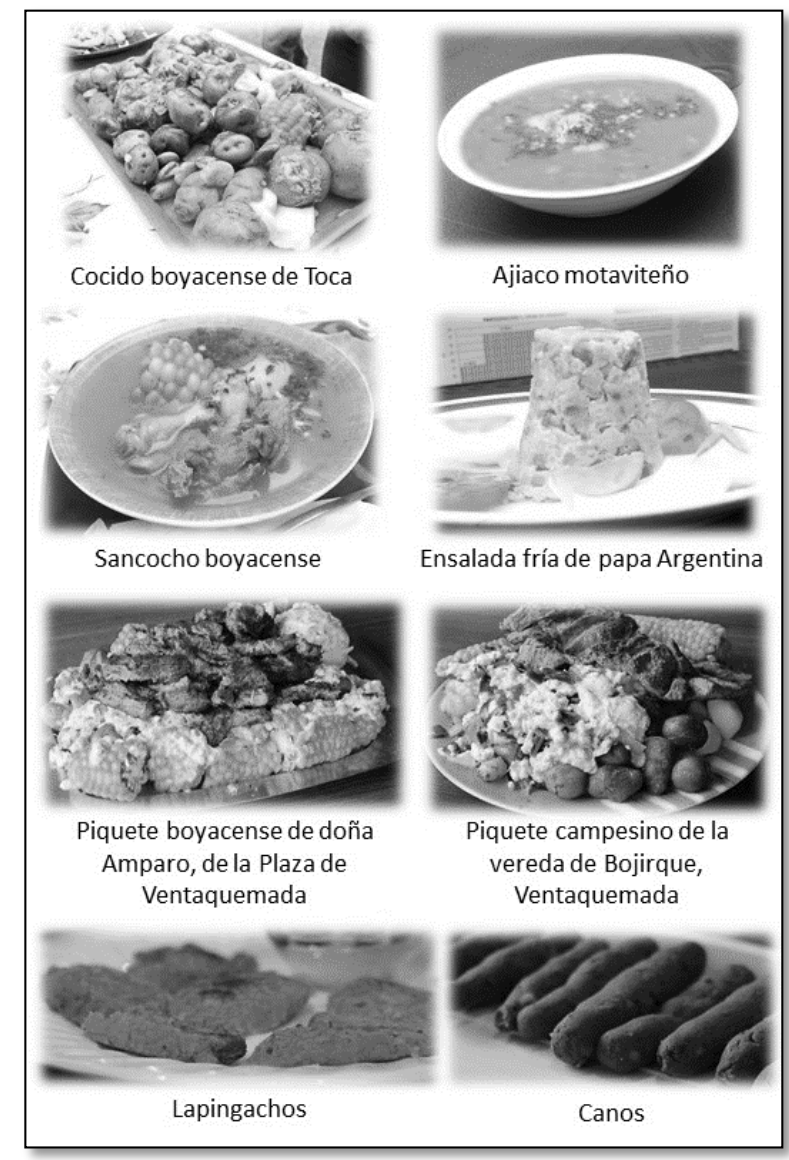

Figura 2. Recetas elaboradas con papas nativas.

Como se mencionó anteriormente, la mayoría de las recetas fueron realizadas con técnicas de cocción húmedas con tiempo controlado, to cual es importante, considerando que la mayoría de las vitaminas de los ingredientes utilizados son hidrosolubles. Al momento de cocinar las papas, es importante seguir un proceso adecuado de cocción e identificar previamente el tipo de preparación que se desea, para así, elegir la variedad de papa más adecuada teniendo en cuenta que cada una tiene características especiales que ayudarán a tener el resultado esperado. Por otro lado, el proceso de cocción podría incidir en el poder antioxidante de las papas nativas, que es una de las bondades de los pigmentos encontrados en este tipo de papas y en el contenido de proteínas del producto final (Moreno - Guerrero et al., 2015; Suárez et al., 2004).

También es importante tener unos procesos de almacenamiento adecuados que permitan tener un producto de alta calidad, ya que al almacenar las papas, estas están sujetas a perder peso y a cambiar su composición química, lo cual puede alterar la calidad de los tubérculos (Crisci, 1992). Por esta razón, siempre hay que almacenarlas en lugares secos y oscuros y desechar producto que esté en mal estado, el cual puede dañar al resto de la materia prima. Por último, es aconsejable no lavarlas hasta que se vayan a utilizar.

Varias de las recetas realizadas en el departamento de Boyacá presentan variaciones según el municipio donde se prepare, como puede verse, por ejemplo, en el caso del cocido y del ajiaco. También, un plato denominado con el mismo nombre, puede variar en su forma de preparación dependiendo de la persona y de los ingredientes que se tengan a la mano. Para el caso de las recetas aquí mencionadas, la mayoría de los ingredientes utilizados son 
comercializados en la mayor parte del territorio colombiano, a excepción de las papas nativas. El cocido, o también conocido como piquete en algunos municipios de la región, fue el plato insignia en las preparaciones realizadas por los participantes. Este, es un plato que inicia con un guiso y posteriormente es aromatizado con diferentes hierbas, se van agregando los ingredientes en orden de cocción y finaliza, en muchos casos, con la adición de leche o cuajada. Aunque algunos de los platos pueden tener una mejora gastronómica, las recetas se mantuvieron tal y como fueron preparadas pues la intención era divulgar las recetas tal cual se preparan en la región.

Según las respuestas dadas por los participantes al momento de entrevistarlos, la mayoría de las recetas aquí presentadas fueron aprendidas de sus padres o abuelos y muchos de ellos mantienen la tradición en la forma de prepararlos y en el uso de los ingredientes y utensilios. Incluso, algunos recordaban con nostalgia cuando sus padres realizaban estos platos en estufas de leña y ollas de barro con cucharones de madera. Por otro lado, también se encontró que algunas de las preparaciones eran creaciones de los participantes y manifestaban haberlas comenzado a preparar, no hace mucho tiempo.
Para tener una buena receta, es importante iniciar con un excelente producto como lo son las papas nativas, un producto $100 \%$ colombiano, pero que, paradójicamente, son desconocidas por gran parte de la población. Actualmente en el mercado colombiano se encuentran más de 30 variedades comerciales y entre estas, las de mayor consumo son la Parda Pastusa, la Pastusa Suprema, la Diacol Capiro, la Ica Única e Ica Puracé, la Criolla, la Tuquerreña y la Roja Nariño (García, 2014), las cuales son utilizadas en la mayoría de las preparaciones culinarias del país; dejando a un lado la comercialización y consumo de las papas nativas.

Actualmente se evidencia un problema nacional como lo es la falta de identidad cultural. En Colombia, se encuentran productos tan valiosos como los cubios, las chuguas, los nabos, las habas, entre otros, que normalmente se asocian a una única preparación, el cocido boyacense. Es una preparación tan antigua pero tan pérdida en el tiempo, que muy pocos restaurantes en Colombia la comercializan y pocas amas de casa la replican. Si esto sucede con productos que muchos conocen desde la infancia, como lo son los mencionados anteriormente, qué se podría decir de las papas nativas, productos visualmente extraños en algunos casos, con texturas 
diversas y poco comercializados, pero con sabores exquisitos.

El resultado obtenido deja ver la importancia de resaltar las papas nativas como un producto nacional de alto valor gastronómico y cultural, y hace pensar en diferentes formas para potencializar su uso. Por un lado, se debe hacer un trabajo con los agricultores, donde sea evidente para ellos las bondades de sembrar este tipo de cultivo. También habría que considerar los canales de distribución de los tubérculos, para que pueda llegar inicialmente a las ciudades de Tunja y Bogotá, que son las ciudades principales cercanas a la región de cultivo y así mismo al resto del país e incluso, fuera de este. Por otro lado, habrá que tener en cuenta la aceptación del público de estas variedades diferentes de papas, las cuales no están acostumbrados a consumir; este es uno de los grandes retos, pues si la población no consume este tipo de productos, es difícil que los agricultores tomen interés en sembrarlas, ya que esto no les dejará muchos ingresos. Una de las alternativas para el uso de las papas nativas es considerarlas como papas élite, considerándolas de mayor valor que las variedades mejoradas que usualmente se comercializan y dándole un potencial que aumentaría sus ventas e ingresos (Sáenz, 2013).
Finalmente, es importante divulgar la existencia de estas papas y la versatilidad que tienen a la hora de cocinarlas. Parte de esto es lo que se ha querido hacer con la presente investigación y para lo cual se publicó un recetario que incluye todas las preparaciones realizadas en las salidas de campo (Chaves et al., 2017). Con esto, se busca dar a conocer al público las papas nativas que se cultivan en el departamento de Boyacá y las diferentes recetas que pueden elaborarse al momento de cocinar. También, se busca crear conciencia sobre la pérdida de la cultura, considerando que la cocina es patrimonio cultural, creemos que debemos trabajar en pro de conservar lo tradicional, o si es el caso, rescatar las recetas que ya han perdido su vigencia en la cocina colombiana. De esta manera, podrá aprovecharse más las papas nativas colombianas, los agricultores al tener un nuevo mercado para sus papas; el consumidor, al tener en sus platos un producto con mejores aportes nutricionales, y finalmente el país, potencializando el uso de un producto autóctono de gran valor. 


\section{CONCLUSIONES}

Con el presente estudio se logró detallar la preparación de 15 platos diferentes, en donde se utilizan las papas nativas del departamento de Boyacá como ingrediente principal. También se encontró que recetas denominadas bajo un mismo nombre pueden variar en su preparación, esto se debe en parte, a las modificaciones que van haciendo las personas de cada municipio, sea por preferencias personales o también por los ingredientes que tengan a la mano.

Las papas nativas son productos muy valiosos para la gastronomía colombiana y pese a su gran valor, estas no son muy conocidas por la población, por lo cual son poco consumidas y comercializadas. Con la presente investigación se logró evidenciar la versatilidad de estas papas al momento de cocinarlas, ya que pueden ser preparadas de diversas maneras y ser utilizadas en diferentes preparaciones, muchas de estas transmitidas de padres a hijos y otras elaboradas por autonomía e ingenio de los participantes.

Se resalta la importancia de ahondar más en el conocimiento de las papas nativas, incentivando su siembra, uso y comercialización, con el fin de rescatar y conservar la gastronomía como patrimonio cultural.

\section{REFERENCIAS BIBLIOGRÁFICAS}

Chaves, J. A., Marín, L. A. \& Quilaguy, D. M. Recetario boyacense con papas nativas Recetas elaboradas con papas nativas colombianas en los municipios de Jenesano, Motavita, Toca, Ventaquemada y Villa de Leyva del Departamento de Boyacá, Colombia. Primera edición. Colombia: Fondo editorial Areandino, 2017.

Crisci, C. Almacenamiento de papa. (1992). Disponible en:
http://www.inia.uy/Publicaciones/Docume ntos\%20compartidos/111219220807120 127.pdf. Consultado: julio de 2016.

García, J. M. Consumo y Mercadeo de la papa en Colombia. (2014). XXVI Congreso Asociación Latinoamericana de la Papa ALAP - Papa, alimento ayer, hoy y siempre. Bogotá, Colombia: Asociación Latinoamericana de la Papa. p. 74-77.

Gutiérrez, R. Papas nativas desafiando al cambio climático. (2008). Disponible en: 
http://www.solucionespracticas.org.pe/De scargar/148/1031. Consultado: julio de 2017.

Hernández, C., Tamayo, A. L., Castro, D. \& Muñoz, I. Tendencias gastronómicas predominantes en la producción de revistas científicas de lberoamérica. (2016). Disponible en: http://www.redalyc.org/articulo.oa?id=104 44319009. Consultado: noviembre de 2016.

Lasso, G. Sostenibilidad rural: el regreso de las papas nativas. (Sin fecha). Disponible en:

http://www.corporacionpba.org/portal/con tent/sostenibilidad-rural-el-regreso-delas-papas-nativas. Consultado: junio de 2017.

Mejía, L. S., Mejía, S. E. \& Bravo, M. Tendencias gastronómicas: La encrucijada entre lo tradicional y lo innovador. (2014). Disponible en: http://web.uaemex.mx/Culinaria/ocho_ne/ PDF\%20finales\%208/Tendencias_gastro nomicas.pdf. Consultado: noviembre de 2016.

Ministerio de Agricultura y Desarrollo Social MINAGRICULTURA. Cadena de papa, indicadores e instrumentos. (2016). Disponible en: https://www.google.com.co/url?sa=t\&rct=i
$\& \mathrm{q}=\&$ esrc $=s \&$ source $=$ web $\& c d=6 \& c a d=r j a$ \&uact=8\&ved=0ahUKEwjeira7u LUAhXL TCYKHQbBBgQQFggzMAU\&url=https\% $3 \mathrm{~A} \% 2 \mathrm{~F} \% 2 \mathrm{Fsioc}$.minagricultura.gov.co\%2 FPapa\%2FDocumentos\%2F002\%2520$\% 2520$ Cifras $\% 2520$ Sectoriales $\% 2 F 002 \%$ $\underline{2520-}$

\%2520Cifras\%2520Sectoriales\%2520 \%25202016\%2520Junio.pptx\&usg=AFQj CNFa9ks7II5oFTo1MsLbJniZyliUgw. Consultado: julio de 2017.

Monteros, C., Navarrete, M. \& Reinoso, I. Las papas nativas en la gastronomía andina. Redescubriendo los sabores y texturas de las papas nativas. (2011). Disponible en: http://www.iniap.gob.ec/nsite/images/doc umentos/Las\%20papas\%20nativas\%20e n\%20la\%20gastronom\%C3\%ADa\%20an dina. Pdf. Consultado: junio de 2017.

Moreno, J. D., Cerón, M. d. S. \& Valvuena, R. I. Papas nativas colombianas, catálogo de 60 variedades. Primera edición. Colombia: Corpoica, 2010.

Moreno - Guerrero, C., Andrade - Cuvi, M. J., Oña - Pillajo, G., Llumiquinga Hernández, T. \& Concellón, A. Efecto de la cocción sobre la composición química y capacidad antioxidante de papas nativas (Solanum tuberosum) del Ecuador. (2015). Disponible en: http://www.agrocalidad.gob.ec/revistaecu 
adorescalidad/index.php/revista/article/vi ew/18/34. Consultado: julio de 2017.

Moreno, J. D. \& Valbuena, I. Colección central colombiana de papa. La mayor riqueza de variabilidad genética para el mejoramiento del cultivo de papa en Colombia. (2006). Disponible en: https://books.google.com.co/books?id=Z G3TVxdnyGUC\&pg=PA24\&lpg=PA24\&d $\mathrm{q}=$ germoplasma+de+papa+en+colombia \&source=bl\&ots=h_Mzpf0QEU\&sig $=u k X$ G6OE0N2Ds4d6YyZxJAjmv5WE\&hl=es\& $\mathrm{sa}=\mathrm{X} \&$ redir_esc $=\mathrm{y} \# \mathrm{v}=$ onepage $\& \mathrm{q}=$ germo plasma\%20de\%20papa\%20en\%20colom bia\&f=false. Consultado: noviembre de 2016.

Navarro, T. Yanine, Villamizar C. Nidia, Duran O. Daniel S., (2013). Caracterización de la polifenoloxidasa en tres variedades de papa (Tuberosum solanum L.) mínimamente procesada y su incidencia en el color, Revista @limentech, Ciencia y Tecnología Alimentaria, ISSN 1692-7125. pp. 5 -12, Vol. $13, N^{\circ} 1$.

Riascos, S. Estructura de los Costos de Producción en el Cultivo de la Papa. (Sin fecha). Revista Fedepapa No. 41.

Disponible

en:

https://www.fedepapa.com/. Consultado: julio de 2017.

Rodríguez, P. Belisario, Maldonado M. Lida Y., Caballero P. Luz A., Evaluación de la aplicación de herramientas de mejora en la industria alimentaria de Norte de Santander, Revista @limentech, Ciencia y Tecnología Alimentaria, pp. 85 -90, Vol. 13, N¹. Año 2013.

Sáenz, M. C. Las nativas como alternativa. (2013). Disponible en: https://es.scribd.com/document/1773563 39/Las-papas-nativas-como-alternativa. Consultado: noviembre de 2016.

Suárez, P., Rodríguez, E. M. \& Díaz, C. Cambios en el valor nutritivo de patatas durante distintos tratamientos culinarios. (2004). Disponible en: http://www.redalyc.org/pdf/724/72440405 .pdf. Consultado: julio de 2017. 


\section{AGRADECIMIENTOS}

Los autores del presente artículo, agradecemos especialmente a las personas que participaron en la elaboración de las recetas y a los respectivos líderes de cada preparación; a Olga Yanet Pérez, Eduardo Espitia, Raúl Iván Valbuena, Camilo Arturo Martínez, Cindy Paola Ortiz, Sara Alejandra Liberato y Yenny Consuelo Peña, de Corpoica, quienes apoyaron la realización de las salidas de campo para la recolección de la información; a los docentes y estudiantes del programa profesional en Gastronomía y Culinaria de la Fundación Universitaria del Área Andina, Luis Antonio Marín, Huil Camacho, Andrey Dueñas, Piedad Sierra, William Sánchez, Lorena Gaviria, María Camila Berrio y Sebastián Cubides, quienes participaron en las salidas de campo y en el registro de la información; y por último, a la Dirección Nacional de Investigaciones de la Fundación Universitaria del Área Andina, por financiar el proyecto y apoyar el desarrollo del mismo. 